\title{
Genetic Engineering of Natural Killer Cells for Enhanced Antitumor Function
}

\author{
Simone Mantesso*, Dirk Geerts, Jan Spanholtz and Lucia Kučerová \\ Research and Development, Glycostem Therapeutics, Oss, Netherlands
}

Natural Killer (NK) cells are unique immune cells capable of efficient killing of infected and transformed cells. Indeed, NK cell-based therapies induced response against hematological malignancies in the absence of adverse toxicity in clinical trials. Nevertheless, adoptive NK cell therapies are reported to have exhibited poor outcome against many solid tumors. This can be mainly attributed to limited infiltration of NK cells into solid tumors, downregulation of target antigens on the tumor cells, or suppression by the chemokines and secreted factors present within the tumor microenvironment. Several methods for genetic engineering of NK cells were established and consistently improved over the last decade, leading to the generation of novel NK cell products with enhanced anti-tumor activity and improved tumor homing. New generations of engineered NK cells are developed to better target refractory tumors and/or to overcome inhibitory tumor microenvironment. This review summarizes recent improvements in approaches to NK cell genetic engineering and strategies implemented to enhance NK cell effector functions.

Keywords: natural killer cells, tumor, genetic engineering, transduction, transfection, chimeric antigen receptornatural killer cells, activating receptors, inhibitory receptors

\section{INTRODUCTION}

Natural killer (NK) cells are part of the innate immune system. Discovered more than 40 years ago, they kill virus-infected cells, counteract tumor formation and initiate innate immune responses (1). Lower NK cell counts and reduced cytotoxicity are associated with higher cancer risks $(2,3)$, as NK cells kill aberrant somatic cells with downregulated major histocompatibility complex class I (MHC-I) molecules that escape T-cell scrutiny (1). Immunotherapy is a powerful biological therapy for boosting the patient's immune system, helping it to fight cancer off. Currently, immunotherapy options include compounds like monoclonal antibodies, cancer vaccines, and checkpoint inhibitors, and more recently cellular products like T cells, dendritic cells or NK cells. Early results showed that NK cells can be a safer alternative over T cells due to reduced side effects. Currently however, NK cell ex vivo expansion technologies are laborious, and their persistence in vivo is limited. Genetic engineering is a valuable tool to overcome these limitations and improve NK cells target specificity and cytotoxicity. NK cells were difficult to genetically modify but recently, NK cell engineering has become efficient and reproducible. This review will summarize recent improvements of NK cell engineering and discuss their use in increasing antitumor efficacy and in vivo persistence through improved tumor homing, and higher target specificity and cytotoxicity (Figure 1). 

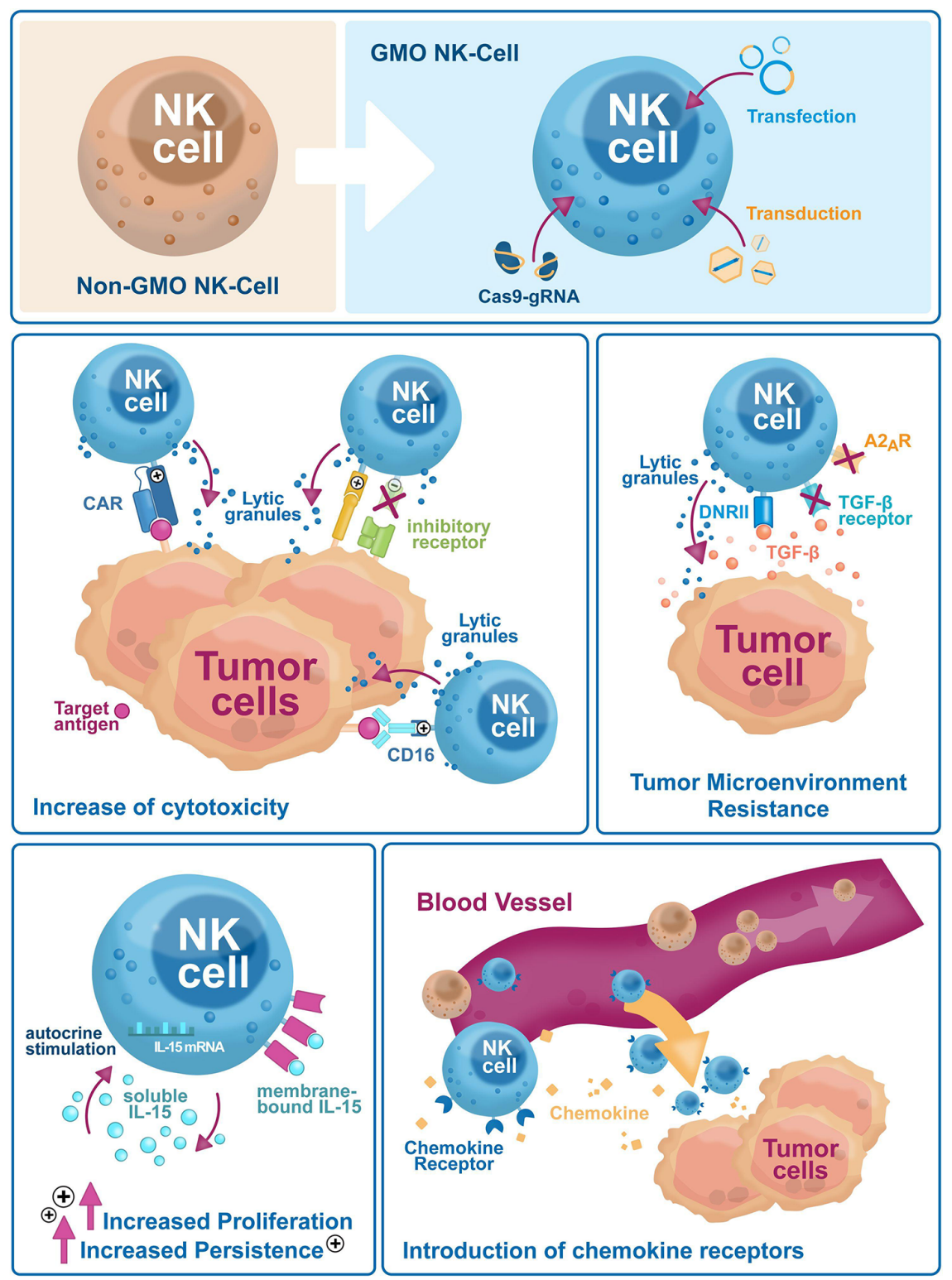

FIGURE 1 | Genetic engineering has broad applications to enhance NK cell-based immunotherapies efficacy against tumor escape mechanisms. Genetic engineering is used on NK cells to improve their cytotoxicity (i.e. exogenous expression of CARs and activating receptors, or selective downregulation of inhibitory receptors), reduce sensitivity to the tumor microenvironment (i.e. downregulation of inhibitory cytokine and small molecule receptors, the introduction of dominantnegative receptors), increase in vivo proliferation and persistence via autocrine cytokines stimulation and tumor homing (i.e. expression of chemokine receptors).

\section{FUNCTIONALITY AND MECHANISM OF ACTION OF NATURAL KILLER CELLS}

NK cells represent the main innate lymphocyte cell type. They mediate both anti-tumor and anti-viral responses. Only antitumor effects will be the subject of this review. NK cells are generally classified as $\mathrm{CD}^{+} 6^{+} \mathrm{CD}^{-}$lymphoid cells and further subdivided into two major subpopulations based on CD56 and CD16 receptor surface expression: $\mathrm{CD} 56^{\mathrm{dim}} \mathrm{CD} 16^{\text {bright }}$ and CD56 ${ }^{\text {bright }} \mathrm{CD} 16^{\text {dim }}$ cells $(4,5)$. Circulating $\mathrm{CD} 56^{\mathrm{dim}} \mathrm{CD} 16^{\text {bright }}$ NK cells are quiescent but become highly cytotoxic upon recognition of target cells, $\mathrm{CD} 56^{\text {bright }} \mathrm{CD} 16^{\text {dim }}$ cells, that reside in secondary lymphoid tissues, constitutively produce cytokines $(6,7)$. NK cells killing ability is tightly regulated by a wide range of inhibitory and activating receptors (1). The most prominent inhibitory receptors are the inhibitory killer-cell immunoglobulin-like receptors (KIRs), that bind polymorphic classical MHC-I molecules (HLA-ABC), universally expressed on healthy cells (8). A major role is also played by the inhibitory heterodimer receptor CD94-NKG2A that binds the non-classical MHC-I molecule HLA-E $(9,10)$. NK cell activating receptors comprise DNAM-1, NKG2D, CD94/NKG2C, CD94/NKG2E, 
natural cytotoxicity receptors (NCRs) like NKp30, NKp44, NKp46, and CD16 (11-14) and activating KIRs. All these recognize specific ligands on the surface of target cells: the CD94/NKG2C and CD94/NKG2E heterodimers recognize HLA-E molecules, the NKG2D receptor recognizes the MHC class-I-chain related proteins A and B (MICA and MICB) and UL16 binding proteins (ULBPs), and DNAM-1 recognizes nectin-2 (CD112) and nectin-like proteins (15, 16). Upon activation, NK cells release lytic granules containing perforin and granzyme B within the immunological synapse to kill target cells (17). NK cells can also exert antibody-dependent cell cytotoxicity (ADCC) by recognizing antibody-coated cells through the low-affinity receptor for the Fc portion of $\operatorname{IgG}_{1}$ antibodies (FcyRIIIa or CD16). Activated NK cells also secrete soluble factors like tumor necrosis factor $\alpha$ (TNF- $\alpha$ ), TNFrelated apoptosis-inducing ligand (TRAIL) and Fas ligand (FasL) to trigger apoptosis in target cells (18). And finally, they secrete interferon $\gamma$ (IFN- $\gamma$ ) (19), growth factors (GM-CSF), immunoregulatory cytokines (IL-15, IL-10, and IL-13) and chemokines $(20,21)$. These cytokines modulate both innate and adaptive immune responses, such as dendritic cell (DC) maturation and $\mathrm{CD} 4{ }^{+}$to $\mathrm{Th} 1 \mathrm{~T}$ cell differentiation, respectively (22-24).

\section{TUMOR CELLS ESCAPE NK CELLS SURVEILLANCE}

NK cells prevent tumor formation and metastases (25). Blood NK cell counts positively correlate with lower risk for cancer development (2), whereas higher tumor tissue NK cell infiltration correlates with improved treatment outcomes (26, 27). This antitumor effect has been comprehensively summarized elsewhere recently (21). However, solid tumors develop escape mechanisms to avoid NK cell recognition (28). They can upregulate MHC-I expression and thereby engage inhibitory NK cell receptors. For example, HLA-E upregulation increases engagement of the NKG2A/CD94 heterodimer in IFN- $\gamma$-stimulated ovarian cancer cell lines, dampening NK cell activity (29). Another resistance mechanism involves the downregulation or shedding of NK cell activating receptor ligands (30). Recently, it was found that NK cells express the immune checkpoint inhibitor PD-1 and that their cytotoxic activity is reduced upon PD-1 engagement in PD-L1 expressing tumors (31). Another recognized key player for tumor cell survival and immune system escape is the tumor microenvironment (TME). The TME comprises tumor-associated non-malignant cells and extracellular matrix components. It secretes suppressive cytokines, like transforming growth factor (TGF)- $\beta$ (32) and IL-10 (33), or suppressive factors like prostaglandin E2 (PGE2) and adenosine $(34,35)$ that prevent NK cell mobilization and target tissue infiltration. Most of these immunosuppressive factors are secreted by tumorassociated cells, mainly regulatory $\mathrm{T}$ cells (T-regs) (36), M2 macrophages and myeloid-derived suppressor cells (MDSCs) (37). Additionally, the TME can be hypoxic by the tumor's high metabolism and poor vascularization, impairing NK cells cytotoxic activity (38). In conjunction, hypoxia favors the selection of the phenotypically most aggressive clones, and tumors become capable of sustained proliferation and metastatic potential that can no longer be controlled by NK cell action (39). Therefore, novel approaches for sustaining NK cells antitumor action are crucial for the development of effective tumor therapy.

\section{NK CELL GENETIC MODIFICATION FOR SUSTAINED FUNCTIONALITY IN CANCER IMMUNOTHERAPY}

Several clinical trials have confirmed the safety profile and efficacy of adoptive NK cells as treatment for hematological malignancies (21). NK cells do not mediate severe toxicities like graft-versus host disease (GvHD) or cytokine release syndrome (CRS) and, therefore, do not require stringent HLA matching $(40,41)$, the bane of T cell therapies. Consequently, NK cells derived from a single donor can be used to treat several patients. This "off-the-shelf application" dramatically improves therapy access and reduces production times and costs. The trials however also highlighted a relatively limited effectivity against solid tumors (21). This is caused by low tumor homing and infiltration, short in vivo persistence and impaired NK cell activity in the cancer patients, this by tumor antigen downregulation and the immunosuppressive TME (28). To overcome these drawbacks, genetic modifications of NK cells has been suggested, and will be discussed in detail in this study. Resting primary NK cells from peripheral blood (PB) or umbilical cord blood (UCB) are difficult to engineer by commonly used approaches, like lentivirus, as low transduction efficiencies were always reported (42). Reduced transduction efficiency rates may be explained by the strong antiviral mechanisms NK cells possess (43). Lentiviral transduction can activate innate immune receptor signaling and trigger NK cell apoptosis. Retrovirus showed higher transduction rates in NK cell lines (especially NK-92) or ex vivo activated and expanded NK cells, mostly PB-NK cells (44) (Table 1). Standard retroviral transduction methods ( $\gamma$-retroviruses), though not known to alter NK cell phenotype and function (45), carry the risk of insertional mutagenesis due to their preference of inserting into active gene promoters, and thereby represent a yet unresolved safety concern, especially if high multiplicity of infection (MOI) rates are used. Besides, NK cell viability after retroviral transduction has been seldom reported and data are scarce. On the other hand, lentiviral transduction methods do not require actively dividing cells, conversely to standard retroviral systems (57). All viral systems are limited in insert size $(<10 \mathrm{~kb})$. All in all, transduction efficiencies remain variable, depending on the NK cell source and MOI (which is seldom reported), and sufficient transduction may require either multiple transductions rounds or post-transduction cell enrichment (Table 1). Alternatively, high transgene expression levels in both primary and ex vivo expanded NK cells $(58,59)$, can be 
TABLE 1 | Genetic modification of NK cells with viral methods.

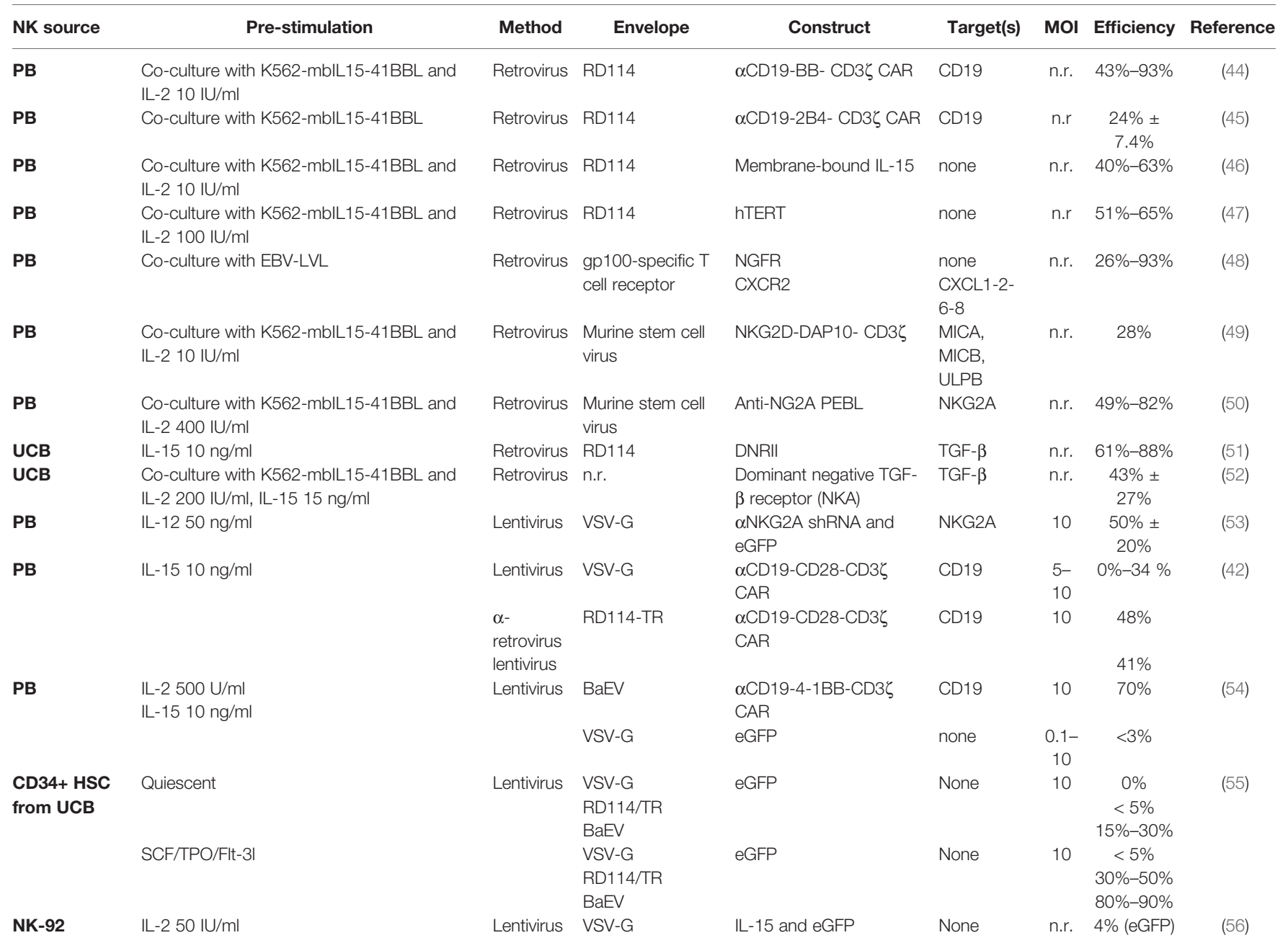

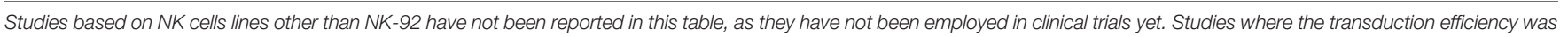

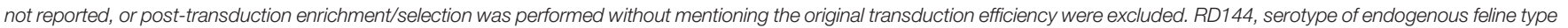
C virus; n.r., not reported; DNRII, dominant negative TGF- $\beta$ RIl; NGFR, Nerve Growth Factor Receptor; PEBL, protein expression blocker.

achieved with transfection methods like electroporation and lipofection, that are not limited in insert size (Table 2). Transgene expression after transfection is however not stable over time, strongly reducing its appeal for clinical applications. Other non-viral genetic integration systems like transposons exist with the advantage to not be limited in insert size. Sleeping Beauty and PiggyBac are the most common and wellcharacterized transposon systems but have so far mainly been tested in T cells, and NK cell studies are still rare and lack safety data. Alternatively, the CRISPR/Cas9 system has been extensively investigated for targeted engineering, with already some studies in NK cells (65). Although CRISPR/Cas9 can be delivered by both viral and non-viral systems, non-viral delivery of a ribonuclear protein (RNP) complex made up by the Cas9 nuclease and the single guide RNA (sgRNA) is preferred, since it limits off-target effects due to viral DNA integration. However, ex vivo non-viral delivery requires optimization, as efficiency is often very limited and viability a concern (61). Of note, CRISPR/ Cas9 can be used to efficiently screen effective synthetic constructs electroporated into $\mathrm{T}$ cells (66), significantly speeding up the discovery of constructs for reprogramming adoptive NK cell functionality and specificity.

Until recently, these limitations delayed NK cell genetic reprogramming for large scale applications compared to T-cells reprogramming. Novel transduction enhancers (e.g. RetroNectin and vectofusin-1) and studies on alternative viral envelopes, mostly genetically modified versions of baboon endogenous retrovirus $(\mathrm{BaEV})$ and $\mathrm{RD} 114$ feline retrovirus glycoprotein (42, 54, 55), contributed to improved NK cell transduction efficiency, rekindling the interest in genetic manipulation of NK cells (Table 1). The BaEV envelope consistently displayed efficient NK cell transduction and might replace the standard VSV-G envelope in NK cell applications. NK cells can efficiently be differentiated from CD34+ hematopoietic stem and progenitor cells (HSPCs) (67) derived from UCB, human embryonic stem cells (hESCs), or induced pluripotent stem cells (iPSCs) $(68,69)$. Both CD34+ HSPCs and iPSCs can be effectively modified with VSV-G, RD114, and BaEV- 
TABLE 2 | Genetic modification of NK cells with non-viral methods.

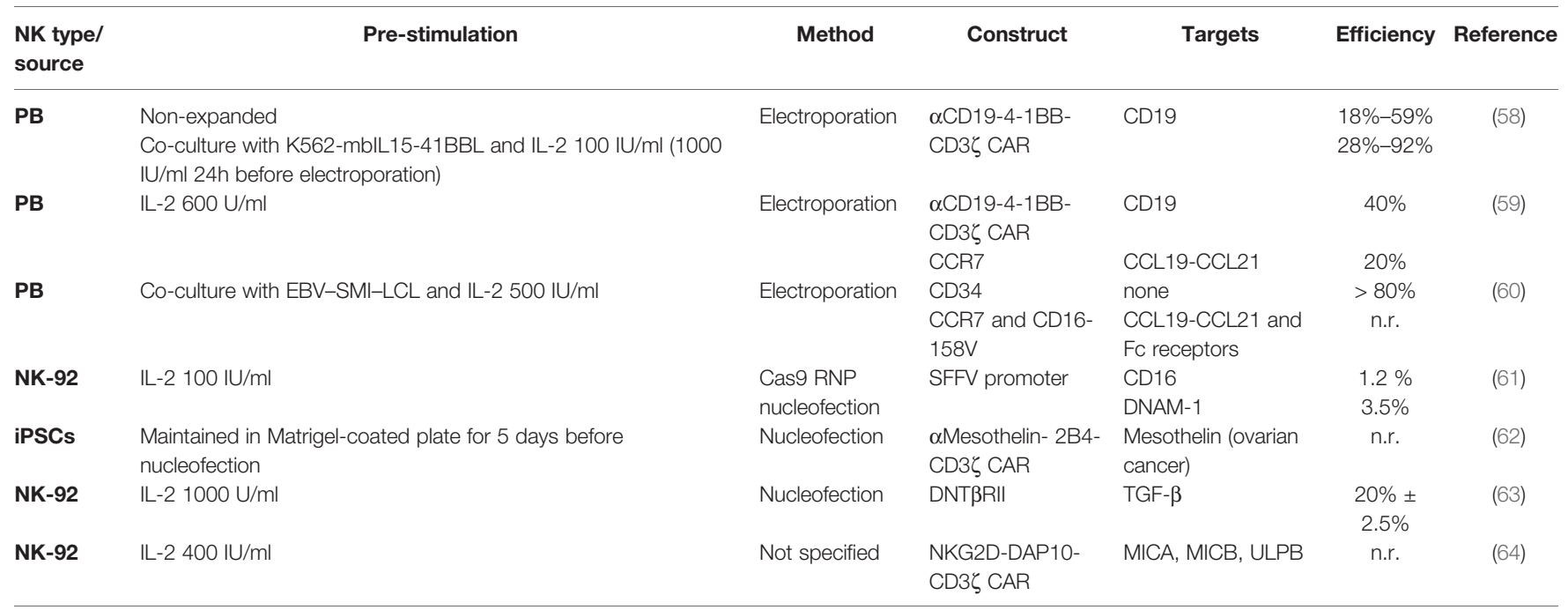

N.r., not reported; DNT $\beta R I I$, dominant negative TGF- $\beta$ RII.

pseudotyped lentiviruses and then differentiated into mature NK cells, and offer another attractive source to generate modified NK cells (55).

In summary, since integrative genetic modification systems are preferred in clinical settings, retrovirus-based genetic engineering has been the established platform to modify NK cells so far. The $\gamma$-retroviral systems are being gradually overtaken by lentiviruses after the discovery of new NK cellspecific envelopes and are set to be the mainstay in NK cell engineering for several years. Studies implementing non-viral strategies have emerged in the last years as well, although they are as of yet mostly limited to pre-clinical settings (Figure 1).

\section{STRATEGIES TO INCREASE TUMOR- SPECIFIC NK CELL CYTOTOXICITY \\ NK Cells Expressing Chimeric Antigen Receptor}

Chimeric antigen receptors (CARs) are antibody-based receptors designed to recognize specific ligands on the surface of target cells. All CAR constructs contain an extracellular antibody single-chain variable fragment ( $\mathrm{scFvs}$ ) fused to a transmembrane region and intracellular immune cell activation domains. The first-generation CAR contained only the intracellular $\mathrm{CD} 3 \zeta$ stimulatory domain of the $\mathrm{T}$ cell receptor (TCR) as activation domain. The second and third generations include one or two additional co-stimulatory domains, respectively (e.g. 4-1BB and/or CD28). CAR technology was first applied on $\mathrm{T}$ cells and although CAR-T cells exhibited strong antitumoral clinical responses, their clinical application is severely curtailed by severe toxicities (GvHD and CRS). This allows only autologous applications for CAR-T cells, which decreases the speed of intervention (negatively affecting therapeutic outcomes), increases production costs and thus decreases treatment accessibility. In addition, $\mathrm{T}$ cells for autologous use are necessarily derived from heavily pre-treated patients, which impinges on their functionality. CAR-NK cells, however, can be derived from allogeneic sources, without apparently causing neither GvHD nor CRS in the recipient, potentially related to their short in vivo persistence and lack of clonal expansion. Off-the-shelf CAR-NK cells can, therefore, have a huge advantage in terms of manufacturing time, costs and accessibility. Besides, allogeneic NK cells are derived from healthy patients and thus retain their normal activity. This allows CAR-NK cells to still exert their anti-tumoral effect in case the CAR expression is decreased or lost, in contrast to CAR-T cells. Only a few CAR-NK phase I/II trials have been started in the last years. Most registered clinical trials employ the NK-92 NK cell line, and the CAR constructs used were based on targets developed for CAR-T cells. This knowledge gap is largely caused by the huge success of anti-CD19 CAR-T cells and the vast amount of clinical data available from CAR-T cell therapies, and as of yet precludes critical assessment of clinal CAR-NK applications. Currently, two CAR-NK cell trials targeting CD19 for leukemia treatment are ongoing. The first one is at the MD Anderson Cancer Centre of the University of Texas (NCT03056339) and is based on UCB-NK cells transduced with a CD19-targeting scFv, interleukin 15 (to enhance NK cells persistence) and an inducible caspase-9 (iC9) suicide gene as a failsafe mechanism. Preliminary results demonstrated that the approach is safe, despite only partial HLA matching between donor and recipient, and potency is high (seven out of 11 patients achieved complete remission) (70). Of note, CAR.19/ IL-15/iC9-NK cells were detected at low levels up to 12 months after the beginning of the treatment, whereas they normally disappear within 2 weeks. Unfortunately, no data about exhaustion is reported, and the influence of pre-conditioning treatment could not be established. The second trial (NCT02892695) is led by PersonGen BioTherapeutics (Suzhou, China), and is based on the NK-92 cell line transduced with a 
third-generation CAR (4-1BBL-CD28-CD3 $\zeta$ co-stimulatory domains). A third phase I study targeting CD19 with haploidentical PBNK cells for B-ALL treatment has been completed (NCT00995137), but no results are available so far. The NK-92 cell line is employed in most of the other phase I/II studies, targeting CD7 (NCT02742727) for lymphoma and leukemia, CD33 for the treatment of Acute Myeloid Leukemia (NCT02944162), HER2 against glioblastoma (NCT03383978) and Mucin-1 (MUC1) in MUC1-positive relapsed or refractory solid tumor-like colorectal carcinoma (CRC) and gastric carcinoma. These and other trials are listed and summarized in Table 3. Efforts directed against multiple myeloma, with CD138 (71) and SLAMF7 (72) as main targets, are still in the preclinical phase. CAR-NK cells targeting solid tumors are now also being explored in preclinical settings. For this, NK cell-specific costimulatory domains are being explored to replace T-cell specific domains in an attempt to increment NK cell-specific activation. Two promising approaches target the prostate stem cell antigen (PSCA), highly expressed on primary prostate tumors and metastases (73), and the epidermal growth factor type III (EGFRvIII), expressed on glioblastoma cells, using the DNAXactivation protein (DAP)12 stimulatory domain instead of one of the commonplace T-cell domains like CD3 $\zeta(74,75)$. DAP12 expression in NK cells induces NKG2C and NKp44 expression upon stimulation. Another group generated an NKG2D-DAP10CD3 $\zeta$ construct (49). The DAP10 domain induces NK cell activation upon phosphorylation through NKG2D-mediated ligand binding $(76,77)$. Although NKG2D is technically not an $\mathrm{scFv}$, the construct is referred to as a CAR, because of its receptor structure. Recently, the NKG2D transmembrane domain has been combined with an anti-mesothelin $\mathrm{scFv}$ and the NK cellspecific signaling domain $2 \mathrm{~B} 4$ and $\mathrm{CD} 3 \zeta$ to target ovarian cancers (62). This construct, cloned in a PiggyBac transposon system, was expressed on iPSC-NK cells, and showed improved specificity and cytotoxicity against the mesothelin-expressing ovarian cancer cell line A1847 and in an ovarian cancer xenograft mouse model. In this mouse model, CAR-iPSC-NK activity was compared with an anti-mesothelin CAR-T expressed in primary $\mathrm{T}$ cells and showed similar anti-tumor activity but significantly lower toxicity and prolonged survival. Of note is also a platform that combines NK cell and T cell advantages, by expressing TCR-CAR chimeric constructs on the NK cell surface (78). Recently, the biotech companies Glycostem Therapeutics and Zelluna Immunotherapies announced a partnership to further develop this field.

\section{NK Cells With Downregulated Inhibitory Receptors}

Cancer cells can throttle immune responses by stimulating key regulators on the surface of immune effector cells known as inhibitory checkpoint molecules. Identification and targeting of inhibitory checkpoints significantly boost immune responses and is therefore of major interest in cancer immunotherapy. During ex vivo expansion of $\mathrm{NK}$ cells, some inhibitory receptors, like NKG2A, are still highly expressed (79-81), suggesting a critical role in $\mathrm{NK}$ cell maturation. On the other end, inhibitory receptors curb NK cell cytotoxic activity and reduce therapeutic efficacy in clinical settings. Indeed, hyporesponsive NKG2A-expressing NK cells are prominent within the TME, thus stressing the importance of this receptor in reducing NK cells activity (82). NKG2A dimerizes with CD94 to bind HLA-E molecules loaded with tumor peptides. While HLA-E surface expression in tumor cells is very weak, IFN- $\gamma$ produced by NK cells can cause its overexpression $(29,50,83)$. Once the peptide/ HLA-E complex is stabilized and binds NKG2A, NK cell activity is dampened. In contrast, RNAi-mediated inhibition of NKG2A expression by shRNA improved NK cell in vitro activity against an HLA-E expressing B-lymphoblastoid cell line (53). Notably, NK cell cytotoxicity was also enhanced against the AML-derived, HLA-E-negative cell line K562. This increased HLA-E

TABLE 3 | Ongoing clinical trials with CAR-NK cells.

\begin{tabular}{|c|c|c|c|}
\hline CAR Target & Condition & Study Phase & NCT \\
\hline CD19/CD22 & B-Cell Lymphoma & 1 & NCT03824964 \\
\hline CD19 & ALL/CLL & $\mid / / 1$ & NCT02892695 \\
\hline CD19 & $\mathrm{ALL}$ & I & NCT00995137 \\
\hline CD33 & AML & $1 / 11$ & NCT02944162 \\
\hline BCMA & MM & $|/| \mid$ & NCT03940833 \\
\hline HER-2 & Glioblastoma & $\|$ & NCT03383978 \\
\hline Mesothelin & Ovarian cancer & I & NCT03692637 \\
\hline PSMA & Prostate cancer & 1 & NCT03692663 \\
\hline ROBO1 & Pancreatic cancer & 1/II & NCT03941457 \\
\hline RОво1 & Solid Tumors & $\mid / / 1$ & NCT03931720 \\
\hline ROBO1 & Solid Tumors & $\mid / / 1$ & NCT03940820 \\
\hline
\end{tabular}

ALL, Acute Lymphocytic Leukemia; AML, Acute Myeloid Leukemia; CLL, Chronic Lymphocytic Leukemia; MM, Multiple Myeloma; NSCLC, Non-small Cell Lung Cancer. 
independent cytotoxicity was probably caused by increased activating NKp30 receptor levels in the NKG2A-negative cell. Unfortunately, these cells have only been tested on a small set of HLA-E positive/negative cell lines. Another group downregulated NKG2A function in PBNKs and NK-92 cells by linking an anti-NKG2A antibody to an endoplasmic reticulumretention domain, and achieved increased cytotoxicity against both HLA-E-positive and -negative cells derived from Ewing's sarcoma, osteosarcoma and AML, as well as prolonged survival in immunodeficient mice expressing HLA-E tumors (50). Blocking of inhibitory receptors represents a feasible approach within the field of biotech industry, although the studies focused on NKG2A only, and their number so far is very limited. Additionally, data comparing NKG2A-negative NK cells and CAR-NK cells activity against the same tumor type are lacking, precluding a comparison between both strategies.

\section{NK Cells With Modified ADCC}

FcyRIII (CD16)-mediated antibody-dependent cell-mediated cytotoxicity (ADCC) plays an important role in tumor clearance. The CD16 isoform expressed on NK cells (CD16a) has two allelic variants with a phenylalanine $(\mathrm{F})$ or valine $(\mathrm{V})$ at amino acid 158, resulting in low (CD16a-158-F/F) and high affinity (CD16a-158-V/V) Fc receptor isoforms. Several studies showed that patients that express the $\mathrm{CD} 16 \mathrm{a}-\mathrm{F} / \mathrm{F}$ variant have better therapy response than patients that are either heterozygous (CD16a-158-V/F) or homozygous for $\mathrm{V}$ upon mAb treatment $(84,85)$. CD16a expression is downregulated upon NK cell activation, mainly due to matrix metalloproteases shedding (86). NK-92 cells, an attractive and cheap source for clinical applications of NK cells, cannot mediate ADCC as they lack CD16 expression. In an attempt to constitutively enhance ADCC activity of NK-92 cells, these cells have been modified to express a CD16a chimera fused to the CD28 and 4-1BB costimulatory domains. Expression of the construct enhanced their cytotoxic activity and restored ADCC activity against CD20positive tumor cells (87). In another attempt, ADCC potency of NK-92 cells was improved by transduction of the high-affinity CD16a-V158 mutant that is resistant to ADAM17-mediated cleavage and shedding $(88,89)$. This cell line, (haNK, developed by NantKwest), showed improved killing capacity compared to PBNK cells from healthy donors and is now in clinical trials against breast cancer (NCT03387085), Merkel cell carcinoma (NCT03853317) and squamous cell carcinoma (NCT03387111). CD16a-158V expression in NK-92 and iPSCs-derived NK cells led to enhanced NK cell activation in the presence of rituximab (90), as well as in PBNKs, although overexpression was transient and lasted no more than 3 days (60). CRISPR/Cas9 has been applied in NK-92 cells by nucleofection to restore endogenous CD16 and DNAM-1 expression by introducing a new promoter upstream the endogenous genes (61). Although this approach was successful, the low nucleofection efficiency and its very high toll on NK cell viability currently preclude its application in ex vivo expanded NK cells, limiting it to NK cell lines capable of autonomous and indefinite growth. As CD16 overexpression markedly improved NK cell activity and cytokine secretion, combinatorial approaches are currently under investigation. For example, NantKwest is employing CAR technology on haNK cells, and has also initiated a phase I clinical study against PD-L1 expressing non-Small Cell Lung Cancer.

\section{NK Cells With Increased Persistence and Proliferation Potential}

In vivo persistence of $\mathrm{NK}$ cells strictly depends on exogenous cytokines. Allogeneic NK cell survival is typically restricted to a couple of weeks, necessitating multiple infusions to achieve therapeutic effects (40). Administration of recombinant IL-2 (rIL-2) in clinical settings resulted in severe toxicities at high doses and activation of inhibitory T-regs at low doses (91). To circumvent these drawbacks, NK-92 cells have been transduced with the IL-2 gene, abolishing NK-92 growth dependence on exogenous IL-2 (92). Transduced NK-92 cells have been tested in nude mice with 3-day-established liver metastases, without exerting side effects after 6 months of treatment. With the discovery that IL-15 has higher potency and lower toxicity than IL-2 (93), the focus has shifted to this cytokine. IL-15 exists in both a soluble and membrane-bound isoforms complexed with IL-15 R $\alpha(94,95)$. NK cells growth dependence on IL-15 has been circumvented by retrovirally transducing PBNK cells with the mbIL-15 membrane-bound isoform, increasing in vivo $\mathrm{NK}$ cell persistence without the need for exogenous IL-2 or IL-15 (46). Stimulatory cytokine signaling has also been recently combined with CAR-NK technology. Briefly, CB-derived NK cells were transduced with a retrovirus encoding a CD19 CAR, soluble IL-15 and the iC9 suicide gene (96). These NK cells had prolonged in vivo survival and were able to control tumor progression significantly better than nonmodified or CD19 CAR-only NK cells. The MD Anderson Cancer Centre phase I clinical study followed, as mentioned above. A CAR construct, targeting the EpCAM carcinoma antigen epithelial cell adhesion molecule, that also encoded IL15 has been transduced into NK-92 cells (56). The IL-15 transgene induced strong proliferation signals, allowing transduced NK-92 cells to grow in the absence of stimulatory cytokines and, additionally, acting as a selection marker for the transduced cells. The CAR gene selectively improved NK cell cytotoxicity against EpCAM-expressing cell lines.

NK cells have low proliferative potential, possibly because of progressive telomere shortening during division cycles. The process can be partially overcome by overexpressing the telomerase reverse transcriptase protein (TERT) responsible for telomere end restoration, normally very lowly expressed in primary cells. Indeed, NK-cell lifespan has already been extended to several months by hTERT overexpression in PBNKs (47). However, transduced PBNKs were still not capable of autonomous growth and proliferation remained dependent on cell-to-cell contacts with feeder cells. Such "immortalized" NK cells can be useful for clinical applications, where large amounts of NK cells need to be injected, and donor availability and/or variability is limiting. Nevertheless, the use of feeder cells in clinical manufacture is not accepted by all regulatory authorities, as it might pose safety concerns for patients. Consequently, feeder cellfree culture systems need to be developed for worldwide 
implementation. Also, hTERT itself can pose safety concerns, as it might drive uncontrolled proliferation, and might require failsafe mechanisms like a suicide gene.

\section{NK Cells With Increased Tumor Homing}

Immune cell homing to and infiltration of tumors is a fundamental prerequisite for effective tumor killing. NK cell ability to infiltrate into the tumor stroma is limited, negatively affecting NK cell therapy efficacy (97). The chemokine receptor CCR7 redirects NK cells preferentially to lymph node-associated chemokine CCL19. CCR7 mRNA transfection into PBNKs by mRNA electroporation improved their in vitro migration towards CCL19 (60). Another group used primary NK cells transduced with retrovirus encoding CXCR2 to improve trafficking towards renal cell carcinoma (48). The NK-like YT cell line was transduced with lentivirus encoding an antiEGFRvIII CAR and the chemokine receptor CXCR4 (75). The CXCR4 receptor promoted specific chemotaxis to glioblastoma cells secreting the CXCL12/SDF-1 $\alpha$ chemokine, while the $\alpha$ EGFRvIII-CAR improved the killing specificity and cytotoxicity. The approach also increased tumor regression and survival in xenograft mouse models.

These studies, although limited and mainly in vitro, demonstrate that NK cell homing to specific tumor sites can improve NK cell-mediated tumor clearance, especially if combined with strategies to enhance NK cell functions after migration to the tumor site.

\section{NK Cells With Increased Resistance to the Tumor Microenvironment}

One of the major immunosuppressive factors within the TME is TGF- $\beta$, produced by various stromal cells, T-regs, MDSCs, and the tumor cells. TGF- $\beta$ interferes with NK cell activation by counteracting several important activating receptors. It downregulates NK cell NKG2D and NKp30 surface expression $(98,99)$ and inhibits CD16-mediated IFN- $\gamma$ production and ADCC in vitro (100). RNAi-mediated knockdown of SMAD3, a TGF- $\beta$ receptor signal transducer, by transduction of NK-92 cells with a lentivirus encoding SMAD3 shRNA increased IFN- $\gamma$, perforin and granzyme $\mathrm{B}$ expression, and enhanced cytotoxicity, increasing tolerance to TGF- $\beta$ signaling both in vitro and in vivo (101). Expression of a chimera consisting of the TGF- $\beta$ receptor type II (TGFBR2) extracellular and transmembrane domain fused to the NKG2D intracellular domain on NK-92 cells caused tolerance to the TGF- $\beta$ signaling and improved chemoattraction to TGF- $\beta$-secreting tumor cell lines (102). Additionally, these NK-92 cells inhibited naïve $\mathrm{CD}^{+} \mathrm{T}$ cell to T-reg differentiation by IFN- $\gamma$ signaling. A dominant-negative mutant form of TGFBR2 (DNRII) has been expressed in CBderived NK cells to block or decrease TGF- $\beta$ signaling. These NK cells did not show downregulation of activating receptors NKG2D and DNAM-1 or of granzyme B and perforin upon TGF- $\beta$ stimulation (51). The same group further created an improved version of the DNRII receptor (renamed "NKA" receptor) by fusing its extracellular domain to DAP12, providing NK cell activating signals upon TGF- $\beta$ stimulation. CB-derived NK cells expressing NKA had enhanced cell cytotoxic activity and persistence against neuroblastoma both in vitro and in vivo (52). A similar approach with the DNRT $\beta I$ receptor in NK-92 cells increased their resistance to TGF- $\beta$ signaling, potentiating antitumor activity in an in vivo lung cancer murine model (63).

Adenosine is emerging as another key negative regulator of NK cells within the TME. Adenosine signals via the $A_{2 A} R$ receptor and limits $\mathrm{NK}$ cell maturation, negatively affecting their proliferation and tumor control (35). Blockage of $\mathrm{A}_{2 \mathrm{~A}} \mathrm{R}$ with an inhibitor caused anti-metastatic effects in breast cancer and melanoma mouse models (103). mAb-mediated inhibition of CD73 ectonucleotidase, one of the key enzymes responsible for extracellular adenosine synthesis, in combination with NKG2D CAR NK-92 cells generated using a PiggyBac transposon system, improved control of CD73-positive tumors (64). The combinatorial approach has been tested against cell lines of prostate cancer (PC3), lung carcinoma (A549) and glioblastoma (GBM43 and GBM10) expressing high levels of CD73 and a xenograft mouse model of lung carcinoma with A549 cells. Although the mAb-CAR combined approach did not dramatically improve anti-tumor activity of the NK-92 cells, the study is important as a first demonstration that stable CAR integration with a non-viral system in NK cells is feasible, and can be extended to other constructs.

\section{FUTURE PERSPECTIVES AND CONCLUDING REMARKS}

NK cell trials have been ongoing for several years by now, demonstrating the safety and efficacy of NK cell-based immunotherapies, especially against hematological malignancies. So far, efficacy against solid tumors is limited, and requires additional technology. NK cells were quite refractory to standard genetic manipulation techniques, resulting in major delays of the first clinical trials with genetically engineered NK cell. Substantial improvements in the last years has led to the first trials. Nonetheless, challenges remain. Viral-based genetic manipulation of NK cells is currently the gold standard to stably express exogenous genes, but transduction efficiency and transgene expression levels are still variable, requiring multiple transductions rounds or posttransduction enrichment. Besides, insertional mutagenesis needs continued safety monitoring. Non-viral delivery methods are still far from routine implementation, as the efficiency and viability can be very low, and the transgene expression can be transient. Low efficiencies are not a great disadvantage with immortalized cells like NK-92, as these can be enriched and then grown indefinitely. But ex vivo expanded NK cells have a limited proliferation potential and enter senescence relatively soon (47). For these cells, highly effective engineering strategies are much more important.

As mentioned above, NK cell efficacy against solid tumors is limited compared to hematological malignancies. Introduction of CAR constructs into NK cells, restoring ADCC functions and/ or downregulation of inhibitory receptors can dramatically 
potentiate their effector functions, helping the patient's immune system in eradicating the disease. The need for NK-specific CAR constructs is now widely recognized, as T-cell-based CARs have reduced activation potentials compared to the former ones, and high-throughput screening techniques will be essential for their identification (66). The limited proliferation potential of NK cells usually does not allow them to persist longer than 2-3 weeks after injection in vivo. Furthermore, homing to tumor sites is often hampered by the TME. Consequently, a highly cytotoxic potential could be relatively limited if NK cells do not persist long enough to eradicate malignant cells or home in on tumor sites. Early efforts in providing NK cells with stimulatory cytokines prolonging half-life like IL-15 are encouraging and worth being further developed, especially in combination with CARs or other activating receptors. Similarly, increasing expression of chemokine receptors on the NK cell surface improves NK cells targeted trafficking and tumor eradication, paving the way for combinatorial strategies. Many solid tumors are difficult to target also because they are encapsulated and protected by a thick layer of extracellular matrix (ECM), a mesh made up mainly by insoluble proteins like type IV collagen and heparan sulphate proteoglycans (HSPGs), that reduce the infiltration abilities of

\section{REFERENCES}

1. Morvan MG, Lanier LL. NK cells and cancer: you can teach innate cells new tricks. Nat Rev Cancer (2016) 16(1):7-19. doi: 10.1038/nrc.2015.5

2. Imai K, Matsuyama S, Miyake S, Suga K, Nakachi K. Natural cytotoxic activity of peripheral-blood lymphocytes and cancer incidence: an 11-year follow-up study of a general population. Lancet (2000) 356(9244):1795-9. doi: 10.1016/s0140-6736(00)03231-1

3. Roder JC, Haliotis T, Klein M, Korec S, Jett JR, Ortaldo J, et al. A new immunodeficiency disorder in humans involving NK cells. Nature (1980) 284(5756):553-5. doi: 10.1038/284553a0

4. Nagler A, Lanier LL, Cwirla S, Phillips JH. Comparative studies of human FcRIII-positive and negative natural killer cells. J Immunol (1989) 143 (10):3183-91.

5. Jacobs R, Hintzen G, Kemper A, Beul K, Kempf S, Behrens G, et al. CD56bright cells differ in their KIR repertoire and cytotoxic features from CD56dim NK cells. Eur J Immunol (2001) 31(10):3121-6. doi: 10.1002/ 1521-4141(2001010)31:10<3121::Aid-immu3121>3.0.Co;2-4

6. Ferlazzo G, Thomas D, Lin SL, Goodman K, Morandi B, Muller WA, et al. The abundant NK cells in human secondary lymphoid tissues require activation to express killer cell Ig-like receptors and become cytolytic. J Immunol (2004) 172(3):1455-62. doi: 10.4049/jimmunol.172.3.1455

7. Fehniger TA, Cooper MA, Nuovo GJ, Cella M, Facchetti F, Colonna M, et al. CD56bright natural killer cells are present in human lymph nodes and are activated by T cell-derived IL-2: a potential new link between adaptive and innate immunity. Blood (2003) 101(8):3052-7. doi: 10.1182/blood-2002-092876

8. Karlhofer FM, Ribaudo RK, Yokoyama WM. MHC class I alloantigen specificity of Ly-49+ IL-2-activated natural killer cells. Nature (6381) 1992) 358:66-70. doi: 10.1038/358066a0

9. Lee N, Llano M, Carretero M, Ishitani A, Navarro F, López-Botet M, et al. HLA-E is a major ligand for the natural killer inhibitory receptor CD94/ NKG2A. Proc Natl Acad Sci U S A (1998) 95(9):5199-204. doi: 10.1073/ pnas.95.9.5199

10. Braud VM, Allan DS, O'Callaghan CA, Soderstrom K, D'Andrea A, Ogg GS, et al. HLA-E binds to natural killer cell receptors CD94/NKG2A, B and C. Nature (1998) 391(6669):795-9. doi: 10.1038/35869

11. Vitale M, Bottino C, Sivori S, Sanseverino L, Castriconi R, Marcenaro E, et al. NKp44, a Novel Triggering Surface Molecule Specifically Expressed by
NK cells (104). Heparanase is upregulated in activated NK cells, improving migration within tumor stroma and playing an important role in reducing tumor growth and metastases (104). To conclude, understanding NK cell biology is another key factor that will help to improve genetic engineering strategies and overcome tumor resistance mechanisms, and allow to fully unleash anti-cancer NK cell potential.

\section{AUTHOR CONTRIBUTIONS}

SM wrote the paper. LK, DG, and JS reviewed the paper. All authors contributed to the article and approved the submitted version.

\section{FUNDING}

This work was supported by a network grant of the European Commission (H2020-MSC-ITN-765104-MATURE-NK) to Glycostem Therapeutics and SM was a fellow in the project, and by a joint grant of the European Commission and EUREKA (E!11764 MODIFY-NK) to Glycostem Therapeutics.

Activated Natural Killer Cells, Is Involved in Non-Major Histocompatibility Complex-restricted Tumor Cell Lysis. J Exp Med (1998) 187(12):2065-72. doi: 10.1084/jem.187.12.2065

12. Bauer S, Groh V, Wu J, Steinle A, Phillips JH, Lanier LL, et al. Activation of NK cells and T cells by NKG2D, a receptor for stress-inducible MICA. Science (1999) 285(5428):727-9. doi: 10.1126/science.285.5428.727

13. Boerman GH, van Ostaijen-ten Dam MM, Kraal KC, Santos SJ, Ball LM, Lankester AC, et al. Role of NKG2D, DNAM-1 and natural cytotoxicity receptors in cytotoxicity toward rhabdomyosarcoma cell lines mediated by resting and IL-15-activated human natural killer cells. Cancer Immunol Immunother (2015) 64(5):573-83. doi: 10.1007/s00262-015-1657-9

14. Sivori S, Vitale M, Morelli L, Sanseverino L, Augugliaro R, Bottino C, et al. p46, a novel natural killer cell-specific surface molecule that mediates cell activation. J Exp Med (1997) 186(7):1129-36. doi: 10.1084/jem.186.7.1129

15. Bottino C, Castriconi R, Pende D, Rivera P, Nanni M, Carnemolla B, et al. Identification of PVR (CD155) and Nectin-2 (CD112) as cell surface ligands for the human DNAM-1 (CD226) activating molecule. J Exp Med (2003) 198 (4):557-67. doi: 10.1084/jem.20030788

16. Tahara-Hanaoka S, Shibuya K, Onoda Y, Zhang H, Yamazaki S, Miyamoto A, et al. Functional characterization of DNAM-1 (CD226) interaction with its ligands PVR (CD155) and nectin-2 (PRR-2/CD112). Int Immunol (2004) 16(4):533-8. doi: 10.1093/intimm/dxh059

17. McCann FE, Vanherberghen B, Eleme K, Carlin LM, Newsam RJ, Goulding $\mathrm{D}$, et al. The size of the synaptic cleft and distinct distributions of filamentous actin, ezrin, CD43, and CD45 at activating and inhibitory human NK cell immune synapses. J Immunol (2003) 170(6):2862-70. doi: 10.4049/ jimmunol.170.6.2862

18. Zamai L, Ahmad M, Bennett IM, Azzoni L, Alnemri ES, Perussia B. Natural killer (NK) cell-mediated cytotoxicity: differential use of TRAIL and Fas ligand by immature and mature primary human NK cells. J Exp Med (1998) 188(12):2375-80. doi: 10.1084/jem.188.12.2375

19. Fauriat C, Long EO, Ljunggren HG, Bryceson YT. Regulation of human NKcell cytokine and chemokine production by target cell recognition. Blood (2010) 115(11):2167-76. doi: 10.1182/blood-2009-08-238469

20. Fehniger TA, Shah MH, Turner MJ, VanDeusen JB, Whitman SP, Cooper MA, et al. Differential cytokine and chemokine gene expression by human NK cells following activation with IL-18 or IL-15 in combination with IL-12: implications for the innate immune response. J Immunol (1999) 162 (8):4511-20 
21. Shimasaki N, Jain A, Campana D. NK cells for cancer immunotherapy. Nature reviews. Drug Discovery (2020) 19(3):200-18. doi: 10.1038/s41573019-0052-1

22. Gerosa F, Baldani-Guerra B, Nisii C, Marchesini V, Carra G, Trinchieri G. Reciprocal activating interaction between natural killer cells and dendritic cells. J Exp Med (2002) 195(3):327-33. doi: 10.1084/jem.20010938

23. Vitale M, Della Chiesa M, Carlomagno S, Pende D, Arico M, Moretta L, et al. NK-dependent DC maturation is mediated by TNFalpha and IFNgamma released upon engagement of the NKp30 triggering receptor. Blood (2005) 106(2):566-71. doi: 10.1182/blood-2004-10-4035

24. Wehner R, Lobel B, Bornhauser M, Schakel K, Cartellieri M, Bachmann M, et al. Reciprocal activating interaction between 6-sulfo LacNAc+ dendritic cells and NK cells. Int J Cancer (2009) 124(2):358-66. doi: 10.1002/ijc.23962

25. Hanna NBR. Definitive evidence that natural killer (NK) cells inhibit experimental tumor metastases in vivo. J Immunol (1981) 127(5):1754-8.

26. Villegas FR, Coca S, Villarrubia VG, Jimenez R, Chillon MJ, Jareno J, et al. Prognostic significance of tumor infiltrating natural killer cells subset CD57 in patients with squamous cell lung cancer. Lung Cancer (2002) 35(1):23-8. doi: 10.1016/s0169-5002(01)00292-6

27. Coca S, Perez-Piqueras J, Martinez D, Colmenarejo A, Saez MA, Vallejo C, et al. The prognostic significance of intratumoral natural killer cells in patients with colorectal carcinoma. Cancer (1997) 79(12):2320-8. doi: 10.1002/(sici)1097-0142(19970615)79:12<2320::aid-cncr5>3.0.co;2-p

28. Nayyar G, Chu Y, Cairo MS. Overcoming Resistance to Natural Killer Cell Based Immunotherapies for Solid Tumors. Front Oncol (2019) 9:51:51. doi: 10.3389/fonc.2019.00051

29. Malmberg KJ, Levitsky V, Norell H, de Matos CT, Carlsten M, Schedvins K, et al. IFN-gamma protects short-term ovarian carcinoma cell lines from CTL lysis via a CD94/NKG2A-dependent mechanism. J Clin Invest (2002) 110 (10):1515-23. doi: 10.1172/JCI15564

30. Siemens DR, Hu N, Sheikhi AK, Chung E, Frederiksen LJ, Pross H, et al. Hypoxia increases tumor cell shedding of MHC class I chain-related molecule: role of nitric oxide. Cancer Res (2008) 68(12):4746-53. doi: 10.1158/0008-5472.CAN-08-0054

31. Hsu J, Hodgins JJ, Marathe M, Nicolai CJ, Bourgeois-Daigneault MC, Trevino TN, et al. Contribution of NK cells to immunotherapy mediated by PD-1/PD-L1 blockade. J Clin Invest (2018) 128(10):4654-68. doi: 10.1172/JCI99317

32. Rouce RH, Shaim H, Sekine T, Weber G, Ballard B, Ku S, et al. The TGFbeta/SMAD pathway is an important mechanism for NK cell immune evasion in childhood B-acute lymphoblastic leukemia. Leukemia (2016) 30 (4):800-11. doi: 10.1038/leu.2015.327

33. Stringaris K, Sekine T, Khoder A, Alsuliman A, Razzaghi B, Sargeant R, et al. Leukemia-induced phenotypic and functional defects in natural killer cells predict failure to achieve remission in acute myeloid leukemia. Haematologica (2014) 99(5):836-47. doi: 10.3324/haematol.2013.087536

34. Holt D, Ma X, Kundu N, Fulton A. Prostaglandin E(2) (PGE (2)) suppresses natural killer cell function primarily through the PGE(2) receptor EP4. Cancer Immunol Immunother (2011) 60(11):1577-86. doi: 10.1007/s00262011-1064-9

35. Young A, Ngiow SF, Gao Y, Patch AM, Barkauskas DS, Messaoudene M, et al. A2AR Adenosine Signaling Suppresses Natural Killer Cell Maturation in the Tumor Microenvironment. Cancer Res (2018) 78(4):1003-16. doi: 10.1158/0008-5472.CAN-17-2826

36. Woo EY, Chu CS, Goletz TJ, Schlienger K, Yeh H, Coukos G, et al. Regulatory CD4(+)CD25(+) T cells in tumors from patients with earlystage non-small cell lung cancer and late-stage ovarian cancer. Cancer Res (2001) 61(12):4766-72.

37. Hoechst B, Voigtlaender T, Ormandy L, Gamrekelashvili J, Zhao F, Wedemeyer $\mathrm{H}$, et al. Myeloid derived suppressor cells inhibit natural killer cells in patients with hepatocellular carcinoma via the NKp30 receptor. Hepatology (2009) 50(3):799-807. doi: 10.1002/hep.23054

38. Chambers AM, Matosevic S. Immunometabolic Dysfunction of Natural Killer Cells Mediated by the Hypoxia-CD73 Axis in Solid Tumors. Front Mol Biosci (2019) 6:60. doi: 10.3389/fmolb.2019.00060

39. Petrova V, Annicchiarico-Petruzzelli M, Melino G, Amelio I. The hypoxic tumour microenvironment. Oncogenesis (2018) 7(1):10. doi: 10.1038/ s41389-017-0011-9
40. Miller JS, Soignier Y, Panoskaltsis-Mortari A, McNearney SA, Yun GH, Fautsch SK, et al. Successful adoptive transfer and in vivo expansion of human haploidentical NK cells in patients with cancer. Blood (2005) 105 (8):3051-7. doi: 10.1182/blood-2004-07-2974

41. Yoon SR, Lee YS, Yang SH, Ahn KH, Lee JH, Lee JH, et al. Generation of donor natural killer cells from CD34(+) progenitor cells and subsequent infusion after HLA-mismatched allogeneic hematopoietic cell transplantation: a feasibility study. Bone Marrow Transplant (2010) 45 (6):1038-46. doi: 10.1038/bmt.2009.304

42. Muller S, Bexte T, Gebel V, Kalensee F, Stolzenberg E, Hartmann J, et al. High Cytotoxic Efficiency of Lentivirally and Alpharetrovirally Engineered CD19-Specific Chimeric Antigen Receptor Natural Killer Cells Against Acute Lymphoblastic Leukemia. Front Immunol (2019) 10:3123. doi: 10.3389/fimmu.2019.03123

43. Sutlu T, Nystrom S, Gilljam M, Stellan B, Applequist SE, Alici E. Inhibition of intracellular antiviral defense mechanisms augments lentiviral transduction of human natural killer cells: implications for gene therapy. Hum Gene Ther (2012) 23(10):1090-100. doi: 10.1089/hum.2012.080

44. Imai C, Iwamoto S, Campana D. Genetic modification of primary natural killer cells overcomes inhibitory signals and induces specific killing of leukemic cells. Blood (2005) 106(1):376-83. doi: 10.1182/blood-2004-124797

45. Altvater B, Landmeier S, Pscherer S, Temme J, Schweer K, Kailayangiri S, et al. 2B4 (CD244) signaling by recombinant antigen-specific chimeric receptors costimulates natural killer cell activation to leukemia and neuroblastoma cells. Clin Cancer Res (2009) 15(15):4857-66. doi: 10.1158/ 1078-0432.CCR-08-2810

46. Imamura M, Shook D, Kamiya T, Shimasaki N, Chai SM, Coustan-Smith E, et al. Autonomous growth and increased cytotoxicity of natural killer cells expressing membrane-bound interleukin-15. Blood (2014) 124(7):1081-8. doi: 10.1182/blood-2014-02-556837

47. Fujisaki H, Kakuda H, Imai C, Mullighan CG, Campana D. Replicative potential of human natural killer cells. Br J Haematol (2009) 145(5):606-13. doi: 10.1111/j.1365-2141.2009.07667.x

48. Kremer V, Ligtenberg MA, Zendehdel R, Seitz C, Duivenvoorden A, Wennerberg E, et al. Genetic engineering of human NK cells to express CXCR2 improves migration to renal cell carcinoma. J Immunother Cancer (2017) 5(1):73. doi: 10.1186/s40425-017-0275-9

49. Chang YH, Connolly J, Shimasaki N, Mimura K, Kono K, Campana D. A chimeric receptor with NKG2D specificity enhances natural killer cell activation and killing of tumor cells. Cancer Res (2013) 73(6):1777-86. doi: 10.1158/0008-5472.CAN-12-3558

50. Kamiya T, Seow SV, Wong D, Robinson M, Campana D. Blocking expression of inhibitory receptor NKG2A overcomes tumor resistance to NK cells. J Clin Invest (2019) 129(5):2094-106. doi: 10.1172/JCI123955

51. Yvon ES, Burga R, Powell A, Cruz CR, Fernandes R, Barese C, et al. Cord blood natural killer cells expressing a dominant negative TGF-beta receptor: Implications for adoptive immunotherapy for glioblastoma. Cytotherapy (2017) 19(3):408-18. doi: 10.1016/j.jcyt.2016.12.005

52. Burga RA, Yvon E, Chorvinsky E, Fernandes R, Cruz CRY, Bollard CM. Engineering the TGFbeta Receptor to Enhance the Therapeutic Potential of Natural Killer Cells as an Immunotherapy for Neuroblastoma. Clin Cancer Res (2019) 25(14):4400-12. doi: 10.1158/1078-0432.CCR-18-3183

53. Figueiredo C, Seltsam A, Blasczyk R. Permanent silencing of NKG2A expression for cell-based therapeutics. J Mol Med (Berl) (2009) 87(2):199210. doi: 10.1007/s00109-008-0417-0

54. Bari R, Granzin M, Tsang KS, Roy A, Krueger W, Orentas R, et al. A Distinct Subset of Highly Proliferative and Lentiviral Vector (LV)-Transducible NK Cells Define a Readily Engineered Subset for Adoptive Cellular Therapy. Front Immunol (2019) 10:2001. doi: 10.3389/fimmu.2019.02001

55. Girard-Gagnepain A, Amirache F, Costa C, Levy C, Frecha C, Fusil F, et al. Baboon envelope pseudotyped LVs outperform VSV-G-LVs for gene transfer into early-cytokine-stimulated and resting HSCs. Blood (2014) 124(8):1221-31. doi: 10.1182/blood-2014-02-558163

56. Sahm C, Schonfeld K, Wels WS. Expression of IL-15 in NK cells results in rapid enrichment and selective cytotoxicity of gene-modified effectors that carry a tumor-specific antigen receptor. Cancer Immunol Immunother (2012) 61(9):1451-61. doi: 10.1007/s00262-012-1212-x 
57. Papayannakos C, Daniel R. Understanding lentiviral vector chromatin targeting: working to reduce insertional mutagenic potential for gene therapy. Gene Ther (2013) 20(6):581-8. doi: 10.1038/gt.2012.88

58. Shimasaki N, Fujisaki H, Cho D, Masselli M, Lockey T, Eldridge P, et al. A clinically adaptable method to enhance the cytotoxicity of natural killer cells against B-cell malignancies. Cytotherapy (2012) 14(7):830-40. doi: 10.3109/ 14653249.2012 .671519

59. Ingegnere T, Mariotti FR, Pelosi A, Quintarelli C, De Angelis B, Tumino N, et al. Human CAR NK Cells: A New Non-viral Method Allowing High Efficient Transfection and Strong Tumor Cell Killing. Front Immunol (2019) 10:957. doi: 10.3389/fimmu.2019.00957

60. Carlsten M, Levy E, Karambelkar A, Li L, Reger R, Berg M, et al. Efficient mRNA-Based Genetic Engineering of Human NK Cells with High-Affinity CD16 and CCR7 Augments Rituximab-Induced ADCC against Lymphoma and Targets NK Cell Migration toward the Lymph Node-Associated Chemokine CCL19. Front Immunol (2016) 7:105. doi: 10.3389/ fimmu.2016.00105

61. Huang R-S, Shih H-A, Lai M-C, Chang Y-J, Lin S. Enhanced NK-92 Cytotoxicity by CRISPR Genome Engineering Using Cas9 Ribonucleoproteins. Front Immunol (2020) 11:1008. doi: 10.3389/ fimmu.2020.01008

62. Li Y, Hermanson DL, Moriarity BS, Kaufman DS. Human iPSC-Derived Natural Killer Cells Engineered with Chimeric Antigen Receptors Enhance Anti-tumor Activity. Cell Stem Cell (2018) 23(2):181-92 e5. doi: 10.1016/ j.stem.2018.06.002

63. Yang B, Liu H, Shi W, Wang Z, Sun S, Zhang G, et al. Blocking transforming growth factor-beta signaling pathway augments antitumor effect of adoptive NK-92 cell therapy. Int Immunopharmacol (2013) 17(2):198-204. doi: 10.1016/j.intimp.2013.06.003

64. Wang J, Lupo KB, Chambers AM, Matosevic S. Purinergic targeting enhances immunotherapy of $\mathrm{CD} 73(+)$ solid tumors with piggyBacengineered chimeric antigen receptor natural killer cells. J Immunother Cancer (2018) 6(1):136. doi: 10.1186/s40425-018-0441-8

65. Afolabi LO, Adeshakin AO, Sani MM, Bi J, Wan X. Genetic reprogramming for NK cell cancer immunotherapy with CRISPR/Cas9. Immunology (2019) 158(2):63-9. doi: 10.1111/imm.13094

66. Roth TL, Li PJ, Blaeschke F, Nies JF, Apathy R, Mowery C, et al. Pooled Knockin Targeting for Genome Engineering of Cellular Immunotherapies. Cell (2020) 181(3):728-44 e21. doi: 10.1016/j.cell.2020.03.039

67. Spanholtz J, Preijers F, Tordoir M, Trilsbeek C, Paardekooper J, de Witte T, et al. Clinical-grade generation of active NK cells from cord blood hematopoietic progenitor cells for immunotherapy using a closed-system culture process. PloS One (2011) 6(6):e20740. doi: 10.1371/journal. pone. 0020740

68. Woll PS, Grzywacz B, Tian X, Marcus RK, Knorr DA, Verneris MR, et al. Human embryonic stem cells differentiate into a homogeneous population of natural killer cells with potent in vivo antitumor activity. Blood (2009) 113 (24):6094-101. doi: 10.1182/blood-2008-06-165225

69. Knorr DA, Ni Z, Hermanson D, Hexum MK, Bendzick L, Cooper LJ, et al. Clinical-scale derivation of natural killer cells from human pluripotent stem cells for cancer therapy. Stem Cells Transl Med (2013) 2(4):274-83. doi: 10.5966/sctm.2012-0084

70. Liu E, Marin D, Banerjee P, Macapinlac HA, Thompson P, Basar R, et al. Use of CAR-Transduced Natural Killer Cells in CD19-Positive Lymphoid Tumors. N Engl J Med (2020) 382(6):545-53. doi: 10.1056/NEJMoa1910607

71. Jiang H, Zhang W, Shang P, Zhang H, Fu W, Ye F, et al. Transfection of chimeric anti-CD138 gene enhances natural killer cell activation and killing of multiple myeloma cells. Mol Oncol (2014) 8(2):297-310. doi: 10.1016/ j.molonc.2013.12.001

72. Chu J, Deng Y, Benson DM, He S, Hughes T, Zhang J, et al. CS1-specific chimeric antigen receptor (CAR)-engineered natural killer cells enhance in vitro and in vivo antitumor activity against human multiple myeloma. Leukemia (2014) 28(4):917-27. doi: 10.1038/leu.2013.279

73. Lam JS, Yamashiro J, Shintaku IP, Vessella RL, Jenkins RB, Horvath S, et al. Prostate stem cell antigen is overexpressed in prostate cancer metastases. Clin Cancer Res (2005) 11(7):2591-6. doi: 10.1158/1078-0432.CCR-04-1842

74. Topfer K, Cartellieri M, Michen S, Wiedemuth R, Muller N, Lindemann D, et al. DAP12-based activating chimeric antigen receptor for NK cell tumor immunotherapy. J Immunol (2015) 194(7):3201-12. doi: 10.4049/ jimmunol.1400330

75. Muller N, Michen S, Tietze S, Topfer K, Schulte A, Lamszus K, et al. Engineering NK Cells Modified With an EGFRvIII-specific Chimeric Antigen Receptor to Overexpress CXCR4 Improves Immunotherapy of CXCL12/SDF-1alpha-secreting Glioblastoma. J Immunother (2015) 38 (5):197-210. doi: 10.1097/CJI.0000000000000082

76. Upshaw JL, Arneson LN, Schoon RA, Dick CJ, Billadeau DD, Leibson PJ. NKG2D-mediated signaling requires a DAP10-bound Grb2-Vav1 intermediate and phosphatidylinositol-3-kinase in human natural killer cells. Nat Immunol (2006) 7(5):524-32. doi: 10.1038/ni1325

77. Wu J, Song Y, Bakker AB, Bauer S, Spies T, Lanier LL, et al. An activating immunoreceptor complex formed by NKG2D and DAP10. Science (1999) 285(5428):730-2. doi: 10.1126/science.285.5428.730

78. Walseng E, Koksal H, Sektioglu IM, Fane A, Skorstad G, Kvalheim G, et al. A TCR-based Chimeric Antigen Receptor. Sci Rep (2017) 7(1):10713. doi: 10.1038/s41598-017-11126-y

79. Spanholtz J, Tordoir M, Eissens D, Preijers F, van der Meer A, Joosten I, et al. High log-scale expansion of functional human natural killer cells from umbilical cord blood CD34-positive cells for adoptive cancer immunotherapy. PloS One (2010) 5(2):e9221. doi: 10.1371/journal.pone. 0009221

80. Denman CJ, Senyukov VV, Somanchi SS, Phatarpekar PV, Kopp LM, Johnson JL, et al. Membrane-bound IL-21 promotes sustained ex vivo proliferation of human natural killer cells. PloS One (2012) 7(1):e30264. doi: 10.1371/journal.pone.0030264

81. Oyer JL, Igarashi RY, Kulikowski AR, Colosimo DA, Solh MM, Zakari A, et al. Generation of highly cytotoxic natural killer cells for treatment of acute myelogenous leukemia using a feeder-free, particle-based approach. Biol Blood Marrow Transplant (2015) 21(4):632-9. doi: 10.1016/j.bbmt. 2014.12.037

82. Mamessier E, Sylvain A, Thibult ML, Houvenaeghel G, Jacquemier J, Castellano R, et al. Human breast cancer cells enhance self tolerance by promoting evasion from NK cell antitumor immunity. J Clin Invest (2011) 121(9):3609-22. doi: 10.1172/JCI45816

83. Marin R, Ruiz-Cabello F, Pedrinaci S, Mendez R, Jimenez P, Geraghty DE, et al. Analysis of HLA-E expression in human tumors. Immunogenetics (2003) 54(11):767-75. doi: 10.1007/s00251-002-0526-9

84. Cartron G, Dacheux L, Salles G, Solal-Celigny P, Bardos P, Colombat P, et al. Therapeutic activity of humanized anti-CD20 monoclonal antibody and polymorphism in IgG Fc receptor FcgammaRIIIa gene. Blood (2002) 99 (3):754-8. doi: 10.1182/blood.v99.3.754

85. Weng WK, Levy R. Two immunoglobulin G fragment C receptor polymorphisms independently predict response to rituximab in patients with follicular lymphoma. J Clin Oncol (2003) 21(21):3940-7. doi: 10.1200/ JCO.2003.05.013

86. Grzywacz B, Kataria N, Verneris MR. CD56(dim)CD16(+) NK cells downregulate CD16 following target cell induced activation of matrix metalloproteinases. Leukemia (2007) 21(2):356-9; author reply 9. doi: 10.1038/sj.leu.2404499

87. Chen Y, You F, Jiang L, Li J, Zhu X, Bao Y, et al. Gene-modified NK-92MI cells expressing a chimeric CD16-BB-zeta or CD64-BB-zeta receptor exhibit enhanced cancer-killing ability in combination with therapeutic antibody. Oncotarget (2017) 8(23):37128-39. doi: 10.18632/oncotarget.16201

88. Jochems C, Hodge JW, Fantini M, Fujii R, Morillon YM, Greiner JW, et al. An NK cell line (haNK) expressing high levels of granzyme and engineered to express the high affinity CD16 allele. Oncotarget (2016) 7(52):86359-73. doi: 10.18632/oncotarget.13411

89. Giles AJ, Hao S, Padget M, Song H, Zhang W, Lynes J, et al. Efficient ADCC killing of meningioma by avelumab and a high-affinity natural killer cell line, haNK. JCI Insight (2019) 4(20):e13068. doi: 10.1172/jci.insight.130688

90. Jing Y, Ni Z, Wu J, Higgins L, Markowski TW, Kaufman DS, et al. Identification of an ADAM17 cleavage region in human CD16 (FcgammaRIII) and the engineering of a non-cleavable version of the receptor in NK cells. PloS One (2015) 10(3):e0121788. doi: 10.1371/ journal.pone. 0121788

91. Ito S, Bollard CM, Carlsten M, Melenhorst JJ, Biancotto A, Wang E, et al. Ultra-low dose interleukin-2 promotes immune-modulating function of 
regulatory $\mathrm{T}$ cells and natural killer cells in healthy volunteers. Mol Ther (2014) 22(7):1388-95. doi: 10.1038/mt.2014.50

92. Nagashima S, Mailliard R, Kashii Y, Reichert TE, Herberman RB, Robbins P, et al. Stable Transduction of the Interleukin-2 Gene Into Human Natural Killer Cell Lines and Their Phenotypic and Functional Characterization In Vitro and In Vivo. Blood (1998) 91(10):3850-61. doi: 10.1182/blood.V91.10.3850

93. Conlon KC, Lugli E, Welles HC, Rosenberg SA, Fojo AT, Morris JC, et al. Redistribution, hyperproliferation, activation of natural killer cells and CD8 $\mathrm{T}$ cells, and cytokine production during first-in-human clinical trial of recombinant human interleukin-15 in patients with cancer. J Clin Oncol (2015) 33(1):74-82. doi: 10.1200/JCO.2014.57.3329

94. Burkett PR, Koka R, Chien M, Chai S, Boone DL, Ma A. Coordinate expression and trans presentation of interleukin (IL)-15Ralpha and IL-15 supports natural killer cell and memory CD8+ T cell homeostasis. J Exp Med (2004) 200(7):825-34. doi: 10.1084/jem.20041389

95. Bergamaschi C, Rosati M, Jalah R, Valentin A, Kulkarni V, Alicea C, et al. Intracellular interaction of interleukin-15 with its receptor alpha during production leads to mutual stabilization and increased bioactivity. $J$ Biol Chem (2008) 283(7):4189-99. doi: 10.1074/jbc.M705725200

96. Liu E, Tong Y, Dotti G, Shaim H, Savoldo B, Mukherjee M, et al. Cord blood NK cells engineered to express IL-15 and a CD19-targeted CAR show longterm persistence and potent antitumor activity. Leukemia (2018) 32(2):52031. doi: $10.1038 /$ leu.2017.226

97. Halama N, Braun M, Kahlert C, Spille A, Quack C, Rahbari N, et al. Natural killer cells are scarce in colorectal carcinoma tissue despite high levels of chemokines and cytokines. Clin Cancer Res (2011) 17(4):678-89. doi: 10.1158/1078-0432.CCR-10-2173

98. Ghiringhelli F, Menard C, Terme M, Flament C, Taieb J, Chaput N, et al. $\mathrm{CD} 4+\mathrm{CD} 25+$ regulatory $\mathrm{T}$ cells inhibit natural killer cell functions in a transforming growth factor-beta-dependent manner. J Exp Med (2005) 202 (8):1075-85. doi: 10.1084/jem.20051511
99. Lee JC, Lee KM, Kim DW, Heo DS. Elevated TGF-betal secretion and downmodulation of NKG2D underlies impaired NK cytotoxicity in cancer patients. J Immunol (2004) 172(12):7335-40. doi: 10.4049/jimmunol. 172.12 .7335

100. Trotta R, Dal Col J, Yu J, Ciarlariello D, Thomas B, Zhang X, et al. TGF-beta utilizes SMAD3 to inhibit CD16-mediated IFN-gamma production and antibody-dependent cellular cytotoxicity in human NK cells. J Immunol (2008) 181(6):3784-92. doi: 10.4049/jimmunol.181.6.3784

101. Wang QM, Tang PM, Lian GY, Li C, Li J, Huang XR, et al. Enhanced Cancer Immunotherapy with Smad3-Silenced NK-92 Cells. Cancer Immunol Res (2018) 6(8):965-77. doi: 10.1158/2326-6066.CIR-17-0491

102. Wang Z, Guo L, Song Y, Zhang Y, Lin D, Hu B, et al. Augmented anti-tumor activity of NK-92 cells expressing chimeric receptors of TGF-betaR II and NKG2D. Cancer Immunol Immunother (2017) 66(4):537-48. doi: 10.1007/ s00262-017-1959-1

103. Mittal D, Young A, Stannard K, Yong M, Teng MW, Allard B, et al. Antimetastatic effects of blocking PD-1 and the adenosine A2A receptor. Cancer Res (2014) 74(14):3652-8. doi: 10.1158/0008-5472.CAN-14-0957

104. Putz EM, Mayfosh AJ, Kos K, Barkauskas DS, Nakamura K, Town L, et al. NK cell heparanase controls tumor invasion and immune surveillance. J Clin Invest (2017) 127(7):2777-88. doi: 10.1172/JCI92958

Conflict of Interest: SM, DG, JP and LK are employed by Glycostem Therapeutics.

Copyright $\odot 2020$ Mantesso, Geerts, Spanholtz and Kučerová. This is an open-access article distributed under the terms of the Creative Commons Attribution License (CC BY). The use, distribution or reproduction in other forums is permitted, provided the original author(s) and the copyright owner(s) are credited and that the original publication in this journal is cited, in accordance with accepted academic practice. No use, distribution or reproduction is permitted which does not comply with these terms. 